\title{
Influencia del voltaje sobre las características reológicas del sabajón
}

\author{
I. Capera U., J. Calderón P., O. Ramos U., O. Restrepo Y. \\ Escuela de Postgrados, Universidad Nacional Abierta y a Distancia, Calle 53 No. 14 - \\ 39. Bogotá, Colombia. Teléfono: 3460088 Fax: 3472556. E-mail: \\ omar.ramos@unad.edu.co
}

\section{Resumen}

Con el objetivo de estudiar el efecto de la diferencia de potencial en los parámetros reológicos, se realizó el estudio del comportamiento reológico del sabajón, sometido a una diferencia de potencial entre 11 voltios y 45 voltios en 11 aplicaciones con un rango de tiempo entre 120 segundos y 630 segundos. Para determinar la significación de los resultados y encontrar los modelos matemáticos adecuados que den respuesta a la correlación de las variables, se realizaron análisis de regresión múltiple para cada una de las variables dependientes con respecto a las independientes. El sabajón es un fluído ligeramente seudoplástico, con índice de flujo promedio de 0,815 y al ser sometido a diferentes voltajes y tiempos de aplicación, dicho índice disminuyó hasta 0,754, haciéndose más seudoplástico El índice de flujo, n, disminuye con la aplicación de voltaje desde 0,815 hasta 0,754 , lo que permite concluir que la seudoplasticidad del sabajón aumenta ligeramente con el tratamiento eléctrico. El índice de consistencia, $\mathrm{k}$, aumenta con la aplicación de voltaje desde 0,112 hasta 0,155 , permitiendo inferir que la viscosidad del fluído (sabajón) aumenta debido a mejor ordenamiento molecular de la muestra tratada.

Palabras Claves: Reología, Propiedades Reológicas, Coloide, Sabajón.

\begin{abstract}
With the objective to study the effect of the difference of potential in the rheologycal parameters, the study of the rheology behaviour of a liquor "sabajón" was made, submissive a difference of potential between 11 volts and 45 volts in 11 applications with a rank of time between 120 second and 630 seconds. In order to determine the meaning of the results and of finding mathematical the models suitable that they give answer to the correlation of the variables, multiple regression analysis for each one of the dependent variables with respect to the independent ones was made. The drink "sabajón" is a slightly pseudoplastic fluid, with flow number average of 0.815 and to the being submissive different voltages and times from application, this index diminished up to 0.754, becoming more pseudoplastic the flow number, $n$, diminishes with the application of voltage from 0.815 to 0.754 , which allows to conclude that the pseudoplasticity of liquor "sabajon" increases slightly with the electrical treatment. The index of consistency, $k$, increases with the application of voltage from 0.112 to 0.155 , allowing to infer that the viscosity of the fluid (sabajon) increases due to better molecular ordering of the treated sample.
\end{abstract}

Key Words: Rheology, Rheologycal Properties, Colloid, Sabajon.

\section{Introducción}

La sedimentación presentada, en el sabajón tiene baja presentación en el mercado, poca aceptación organoléptica; en consecuencia la credibilidad de su calidad se pone en riesgo, bajando el nivel de demanda con afectación económica a las empresas productoras debido a las devoluciones del producto o disminución en la producción. 
Se hace necesario implementar un plan de estabilización del coloide (sabajón) que incida en el mejoramiento del producto. Este incluye, el estudio de los compuestos sedimentables del coloide y plantear una alternativa de solución. El sabajón está compuesto por sustancias mayoritariamente polares, tales como: agua, etanol, proteínas, carbohidratos y compuestos alimenticios orgánicos, los cuales son materiales potencialmente electrorreológicos dentro del coloide. Para determinar el plan de estabilización del sabajón, se tiene en cuenta que este, es un fluído electrorreológico, debido a la polaridad de los componentes de las fases del coloide. El plan de estabilización del sabajón consiste en aplicar al coloide, diferentes voltajes para generar un campo eléctrico, de esta forma producir migración molecular hacia los dos polos, cambiando la estructura del coloide y conseguir un ordenamiento molecular que conlleva a un cambio físico del estado líquido a semisólido.

El ordenamiento molecular ocurre por atracción en el sentido paralelo y repulsión en el sentido perpendicular a la dirección del campo eléctrico, formando cadenas de partículas polarizables de microtamaño, que aumentan, en varios ordenes de magnitud, las propiedades reológicas del fluído, especialmente la viscosidad aparente; dándole al coloide, mayor estabilidad respecto a la muestra sin aplicación de voltaje. La variación de las propiedades reológicas del fluído ocasionadas por la aplicación del voltaje se determina clasificando al fluído, luego cuantificando la viscosidad aparente, esfuerzo de corte, índices de flujo y consistencia, a diferentes gradientes de velocidad de cizallamiento, voltajes y tiempos de aplicación de este.

El trabajo de investigación a nivel laboratorio, busca establecer las características reológicas del sabajón en función de un intervalo de voltaje y tiempo de aplicación, determinados de acuerdo con un diseño factorial multivariable. Donde se formulan dos objetivos:

- Determinar el comportamiento reológico del fluido sabajón, encontrando la relación que existe entre el esfuerzo de corte y el gradiente de velocidad bajo condiciones de aplicación de voltaje y tiempo de exposición constante, para cada ensayo.

- Establecer los modelos matemáticos que permitan determinar el índice de consistencia, el índice de fluidez, la viscosidad aparente y el esfuerzo cortante, para un voltaje aplicado durante un tiempo determinado.

\section{Metodología}

\section{Muestra}

La muestra que se tomó fue Sabajón, el cual, según el decreto 365 de 1994, emanado por el Ministerio de Salud Pública, en el artículo $6^{\circ}$, numeral 6.3, lo clasifica como un aperitivo no vínico adicionado de productos alimenticios orgánicos. El mismo decreto define también como el producto obtenido por mezcla de leche, huevos, azúcar, con adición de alcohol etílico neutro certificado, aguardiente u otros licores y aditivos permitidos por el Ministerio de Salud, con una graduación entre 14 y 20 grados alcoholimétricos.

\section{Variables}

Independientes: voltaje aplicado, medido en voltios, y tiempo de aplicación del voltaje, medido en segundos. 
Dependientes: esfuerzo de corte $(\boldsymbol{\tau})$, viscosidad aparente $(\eta)$, índice de consistencia (k) e índice de flujo (n)

De control o de Restricción: Temperatura $\left(20^{\circ} \mathrm{C}\right)$, intensidad de corriente (3 A) y área de electrodos

\section{Diseño Experimental}

Se estableció el diseño experimental factorial multivariable de $\mathbf{2}^{\mathbf{n}}$, con las variables independientes voltaje y tiempo y las variables dependientes esfuerzo de corte, viscosidad aparente, índice de consistencia e índice de flujo. Donde $n$ corresponde al número de variables independientes y 2 al número de niveles considerados y que para este caso se codifican como $+1 \mathrm{y}-1$.

$$
\text { No. de ensayos }=2^{\mathrm{n}}=2^{2}=4 \text { ensayos. }
$$

Además de estos 4 ensayos se realizaron 3 de nivel central (nivel 0) a fin de estimar el error, y cuatro ensayos de segundo orden, (niveles de $-\sqrt{2}$ y $+\sqrt{ } 2$ ) con el propósito de obtener una mayor confiabilidad en los resultados, para un total de 11 ensayos.

La asignación de los valores de tiempo y voltaje para los diferentes niveles, se realizó arbitrariamente, debido a que no se encuentran referentes bibliográficos para el sabajón. Se estableció el nivel cero como 25 voltios y 420 segundos y los demás valores se obtuvieron teniendo en cuenta una amplitud de 14 voltios y 210 segundos para cada unidad codificada, respectivamente. A continuación se relacionan los valores correspondientes en la tabla 1:

Tabla 1. Factores y Niveles del Diseño Experimental

\begin{tabular}{|c|c|c|c|c|}
\hline Ensayo & $\begin{array}{c}\text { Voltaje } \\
\text { Codificado }\end{array}$ & $\begin{array}{c}\text { Voltaje } \\
\text { volts }\end{array}$ & Tiempo Codificado & $\begin{array}{c}\text { Tiempo } \\
\text { seg }\end{array}$ \\
\hline 1 & -1 & 11 & -1 & 210 \\
\hline 2 & +1 & 39 & -1 & 210 \\
\hline 3 & -1 & 11 & +1 & 630 \\
\hline 4 & +1 & 39 & +1 & 630 \\
\hline 5 & 0 & 25 & 0 & 420 \\
\hline 6 & 0 & 25 & 0 & 420 \\
\hline 7 & 0 & 25 & 0 & 420 \\
\hline 8 & $-1,414$ & 5 & 0 & 420 \\
\hline 9 & 0 & 25 & $+1,414$ & 720 \\
\hline 10 & $+1,414$ & 45 & 0 & 420 \\
\hline 11 & 0 & 25 & $-1,414$ & 120 \\
\hline
\end{tabular}

\section{Procedimiento}

Descripción del Equipo. Para la determinación experimental se utilizó el viscosímetro Rotovisco RV 20, el cual mide el porcentaje de torque $(\% \tau) \mathbf{A}$ y el porcentaje de gradiente de velocidad $(\% \gamma)$ M. Se trata de un viscosímetro de cilindros concéntricos de radio finito. El sistema sensor $\mathrm{NV}$ consta de un cilindro interno y un rotor en forma de campana. Se clasifica como un sistema de sensor cilíndrico coaxial con dos espacios para cizallar las muestras sobre la parte interna y la parte externa del rotor. 
Dependencia de las características reológicas con el tiempo (tixotropía). Con el objeto de determinar si las características reológicas del sabajón dependen del tiempo, se tomó una muestra y se determinó el porcentaje de esfuerzo de corte, a porcentajes de velocidad de cizallamiento consecutivos de: 1,$0 ; 1,6 ; 2,8 ; 4,6 ; 7,7 ; 12,9$ y 21,5 . A la misma muestra se le determinó nuevamente el porcentaje de esfuerzo de corte, tomando los mismos porcentajes de velocidad de cizallamiento, pero en orden descendente.

Aplicación del voltaje. Se tomó para cada ensayo una muestra de sabajón en un vaso de precipitados, se les aplicó el voltaje definido, durante el tiempo correspondiente a cada ensayo, propuestos en el diseño experimental, manteniendo constantes la temperatura, la intensidad de corriente y el área de los electrodos; se determinaron los porcentajes de esfuerzo de corte a porcentajes de velocidad de cizallamiento consecutivas iguales a 1,$0 ; 1,6 ; 2,8 ; 4,6 ; 7,7 ; 12,9$ y 21,5 .

Determinación del gradiente de velocidad, $\boldsymbol{\gamma}$. El gradiente de velocidad se determinó con la siguiente ecuación:

$\begin{array}{ll} & \gamma\left(\mathrm{s}^{-1}\right)=\mathrm{M} \times \% \quad \text { (Ec. 1) } \\ \text { Para el equipo utilizado: } & \mathrm{M}=27 \mathrm{~s}^{-1} \% \gamma \quad \text { (Ec. 2) }\end{array}$

Determinación del esfuerzo de corte, $\tau$. Para calcular el esfuerzo de corte se utilizó la siguiente ecuación:

$$
\begin{array}{ccc} 
& \tau(\mathrm{Pa})=\mathrm{A} \times \% \tau \quad(\text { Ec. } 3) \\
\text { Para el equipo utilizado: } \quad \mathrm{A}=1,78 \mathrm{~Pa} / \% \tau & (\text { Ec. } 4)
\end{array}
$$

Determinación del grado de tixotropía. Para conocer si el sabajón sin aplicación de voltaje presenta comportamiento tixotrópico o reopéctico, se compararon los resultados obtenidos para gradientes de velocidad ascendentes y descendentes.

Aplicando el modelo de Ley de Potencia, donde $\tau=\mathbf{k} \boldsymbol{\gamma}^{\mathbf{n}}$ mediante el uso del software Curvexpert 1.3, se graficaron los valores de $\tau$ Vs. $\boldsymbol{\gamma}$, tanto con gradiente de velocidad ascendente, como con gradiente de velocidad descendente; se calculó el área bajo cada una de las curvas, se estableció la diferencia de las áreas y se dividió por el área mayor para encontrar el \% de tixotropía.

\section{Determinación de los índices de flujo y de consistencia a los diferentes voltajes y} tiempo de aplicación. Se graficó $\tau$ Vs. $\boldsymbol{\gamma}$ para cada voltaje y tiempo de aplicación tomándose el modelo de ley de potencia $\boldsymbol{\tau}=\mathbf{k} \boldsymbol{\gamma}^{\mathbf{n}}$, y estableciéndose la correlación con el software Curvexpert 1.3.

Determinación de la viscosidad aparente, $\boldsymbol{\eta}$. Se calculó punto a punto, dividiendo el esfuerzo de corte $\tau$, entre el gradiente de velocidad $\gamma$. Así, se tiene que para fluidos que cumplen la Ley de Potencia 


$$
\begin{aligned}
\eta & =\mathrm{k} \gamma^{(\mathrm{n}-1)}=\mathrm{k} \gamma^{\mathrm{n}} / \gamma \quad \text { (Ec. 5) } \\
\text { Como } \boldsymbol{\tau} & =\mathbf{k} \boldsymbol{\gamma}^{\mathrm{n}} \quad \text { Entonces: } \eta=\tau / \gamma
\end{aligned}
$$

\section{Resultados}

De los datos primarios, se obtuvieron los valores de esfuerzo de corte, gradiente de velocidad, viscosidad aparente y el grado de tixotropía, según lo indicado en el procedimiento para cada caso.

Tabla 2. Esfuerzo de corte $(\tau)$ y viscosidad aparente $(\eta)$ a diferentes voltajes $(V)$ y tiempos de aplicación (t), para cada gradiente de velocidad $(\gamma)$.

$\gamma=43,21 / \mathrm{s}$

\begin{tabular}{|c|c|c|c|}
\hline Voltaje, $\mathbf{V}$ & Tiempo, $\mathbf{s}$ & $\boldsymbol{\tau}, \mathbf{P a}$ & $\boldsymbol{\eta}(\mathbf{m P a} \mathbf{s})$ \\
\hline 11 & 210 & 2,53 & 58,509 \\
\hline 39 & 210 & 2,74 & 63,454 \\
\hline 11 & 630 & 2,51 & 58,097 \\
\hline 39 & 630 & 2,76 & 63,866 \\
\hline 25 & 420 & 2,67 & 61,806 \\
\hline 25 & 420 & 2,67 & 61,806 \\
\hline 25 & 420 & 2,58 & 59,745 \\
\hline 5 & 420 & 2,55 & 58,921 \\
\hline 25 & 720 & 2,67 & 61,806 \\
\hline 45 & 420 & 2,85 & 65,926 \\
\hline 25 & 120 & 2,69 & 62,218 \\
\hline
\end{tabular}

$\gamma=75,61 / \mathrm{s}$

\begin{tabular}{|c|c|c|c|}
\hline Voltaje, $\mathbf{V}$ & Tiempo, $\mathbf{s}$ & $\boldsymbol{\tau}, \mathbf{P a}$ & $\boldsymbol{\eta}(\mathbf{m P a}$ *s) \\
\hline 11 & 210 & 3,83 & 50,622 \\
\hline 39 & 210 & 4,20 & 55,566 \\
\hline 11 & 630 & 3,83 & 50,622 \\
\hline 39 & 630 & 4,20 & 55,566 \\
\hline 25 & 420 & 4,01 & 52,976 \\
\hline 25 & 420 & 3,99 & 52,741 \\
\hline 25 & 420 & 3,99 & 52,741 \\
\hline 5 & 420 & 3,90 & 51,563 \\
\hline 25 & 720 & 4,08 & 53,918 \\
\hline 45 & 420 & 4,18 & 55,331 \\
\hline 25 & 120 & 4,11 & 54,389 \\
\hline
\end{tabular}

$\gamma=124,21 / \mathrm{s}$

\begin{tabular}{|c|c|c|c|}
\hline Voltaje, $\mathbf{V}$ & Tiempo, $\mathbf{s}$ & $\boldsymbol{\tau}, \mathbf{P a}$ & $\boldsymbol{\eta}\left(\mathbf{m P a}{ }^{*} \mathbf{s}\right)$ \\
\hline 11 & 210 & 5,86 & 47,151 \\
\hline 39 & 210 & 6,27 & 50,448 \\
\hline 11 & 630 & 5,87 & 47,295 \\
\hline 39 & 630 & 6,28 & 50,591 \\
\hline
\end{tabular}




\begin{tabular}{|c|c|c|c|}
\hline 25 & 420 & 6,05 & 48,728 \\
\hline 25 & 420 & 5,98 & 48,155 \\
\hline 25 & 420 & 5,96 & 48,011 \\
\hline 5 & 420 & 5,87 & 47,295 \\
\hline 25 & 720 & 6,11 & 49,158 \\
\hline 45 & 420 & 6,23 & 50,161 \\
\hline 25 & 120 & 6,09 & 49,014 \\
\hline
\end{tabular}

$\gamma=207,91 / \mathrm{s}$

\begin{tabular}{|c|c|c|c|}
\hline Voltaje, $\mathbf{V}$ & Tiempo, $\mathbf{s}$ & $\boldsymbol{\tau}, \mathbf{P a}$ & $\boldsymbol{\eta}(\mathbf{m P a} \mathbf{*})$ \\
\hline 11 & 210 & 8,83 & 42,467 \\
\hline 39 & 210 & 9,29 & 44,693 \\
\hline 11 & 630 & 8,78 & 42,210 \\
\hline 39 & 630 & 9,26 & 44,521 \\
\hline 25 & 420 & 9,02 & 43,408 \\
\hline 25 & 420 & 8,81 & 42,381 \\
\hline 25 & 420 & 8,92 & 42,895 \\
\hline 5 & 420 & 8,74 & 42.038 \\
\hline 25 & 720 & 9,06 & 43,580 \\
\hline 45 & 420 & 9,26 & 44,521 \\
\hline 25 & 120 & 9,08 & 43,665 \\
\hline
\end{tabular}

$\gamma=348,31 / \mathrm{s}$

\begin{tabular}{|c|c|c|c|}
\hline Voltaje, $\mathbf{V}$ & Tiempo, $\mathbf{s}$ & $\boldsymbol{\tau}, \mathbf{P a}$ & $\boldsymbol{\eta}(\mathbf{m P a} \mathbf{s})$ \\
\hline 11 & 210 & 13,23 & 37,971 \\
\hline 39 & 210 & 13,72 & 39,402 \\
\hline 11 & 630 & 13,08 & 37,562 \\
\hline 39 & 630 & 13,69 & 39,300 \\
\hline 25 & 420 & 13,35 & 38,329 \\
\hline 25 & 420 & 13,08 & 37,562 \\
\hline 25 & 420 & 13,35 & 38,329 \\
\hline 5 & 420 & 13,01 & 37,358 \\
\hline 25 & 720 & 13,39 & 38,431 \\
\hline 45 & 420 & 13,74 & 39,453 \\
\hline 25 & 120 & 13,26 & 38,073 \\
\hline
\end{tabular}

$\gamma=\mathbf{5 8 0 , 5} 1 / \mathrm{s}$

\begin{tabular}{|c|c|c|c|}
\hline Voltaje, $\mathbf{V}$ & Tiempo, $\mathbf{s}$ & $\boldsymbol{\tau}, \mathbf{P a}$ & $\boldsymbol{\eta}$ (mPa*s) \\
\hline 11 & 210 & 20,83 & 35,876 \\
\hline 39 & 210 & 20,45 & 35,232 \\
\hline 11 & 630 & 19,58 & 33,730 \\
\hline 39 & 630 & 20,35 & 35,048 \\
\hline 25 & 420 & 19,58 & 33,730 \\
\hline 25 & 420 & 19,49 & 33,576 \\
\hline 25 & 420 & 19,49 & 33,576 \\
\hline
\end{tabular}




\begin{tabular}{|c|c|c|c|}
\hline 5 & 420 & 19,49 & 33,576 \\
\hline 25 & 720 & 19,97 & 34,404 \\
\hline 45 & 420 & 20,29 & 34,956 \\
\hline 25 & 120 & 19,97 & 34,404 \\
\hline
\end{tabular}

Tabla 3. Determinación del grado de tixotropía

\begin{tabular}{|c|c|c|c|}
\hline Gradiente de velocidad & K Pas $^{\mathbf{n}}$ & $\mathbf{n}$ & Área bajo la curva \\
\hline Ascendente & 0,121 & 0,803 & 7614,4 \\
\hline Descendente & 0,103 & 0,828 & 7469,3 \\
\hline
\end{tabular}

Grado de tixotropía $=(7614,6-7469,3) \times 100 / 7614,6=1,90 \%$

Tabla 4. Índices de consistencia y flujo a diferentes voltajes y tiempos de aplicación

\begin{tabular}{|c|c|c|c|c|c|}
\hline $\begin{array}{c}\text { Voltaje } \\
\text { Voltios }\end{array}$ & $\begin{array}{c}\text { Tiem } \\
\mathbf{p o} \\
\mathbf{s}\end{array}$ & $\begin{array}{c}\text { Ecuación } \\
\text { Ley de Potencia }\end{array}$ & $\begin{array}{c}\text { Coeficiente } \\
\text { Correlación } \\
\mathbf{r}\end{array}$ & $\begin{array}{c}\text { Indice de } \\
\text { Flujo } \mathbf{n}\end{array}$ & $\begin{array}{c}\text { Indice de } \\
\text { Consistencia } \\
\mathbf{k} \text { Pa s }\end{array}$ \\
\hline 11 & 210 & $\boldsymbol{\tau}=0,136 \boldsymbol{\gamma}^{\mathbf{0 , 7 5 4}}$ & 0,99999 & 0,754 & 0,136 \\
\hline 39 & 210 & $\boldsymbol{\tau}=0,151 \boldsymbol{\gamma}^{\mathbf{0 , 7 7 1}}$ & 0,99997 & 0,771 & 0,151 \\
\hline 11 & 630 & $\boldsymbol{\tau}=0,135 \boldsymbol{\gamma}^{\mathbf{0 , 7 5 5}}$ & 0,99999 & 0,755 & 0,135 \\
\hline 39 & 630 & $\boldsymbol{\tau}=0,153 \boldsymbol{\gamma}^{\mathbf{0 , 7 6 8}}$ & 0,99998 & 0,768 & 0,153 \\
\hline 25 & 420 & $\boldsymbol{\tau}=0,151 \boldsymbol{\gamma}^{\mathbf{0 , 7 6 5}}$ & 0,99993 & 0,765 & 0,151 \\
\hline 25 & 420 & $\boldsymbol{\tau}=0,144 \boldsymbol{\gamma}^{\mathbf{0 , 7 7 1}}$ & 0,99997 & 0,771 & 0,144 \\
\hline 25 & 420 & $\boldsymbol{\tau}=0,146 \boldsymbol{\gamma}^{\mathbf{0 , 7 7 0}}$ & 0,99990 & 0,770 & 0,146 \\
\hline 5 & 420 & $\boldsymbol{\tau}=0,133 \boldsymbol{\gamma}^{\mathbf{0 , 7 8 3}}$ & 0,99998 & 0,783 & 0,133 \\
\hline 25 & 720 & $\boldsymbol{\tau}=0,146 \boldsymbol{\gamma}^{\mathbf{0 , 7 7 3}}$ & 0,99998 & 0,773 & 0,146 \\
\hline 45 & 420 & $\boldsymbol{\tau}=0,155 \boldsymbol{\gamma}^{\mathbf{0 , 7 6 6}}$ & 0,99997 & 0,766 & 0,155 \\
\hline 25 & 120 & $\boldsymbol{\tau}=0,146 \boldsymbol{\gamma}^{\mathbf{0 , 7 7 3}}$ & 0,99991 & 0,773 & 0,146 \\
\hline 0 & 0 & $\boldsymbol{\tau}=0,121 \boldsymbol{\gamma}^{\mathbf{0 , 8 0 3}}$ & 0,99994 & 0,815 & 0,112 \\
\hline
\end{tabular}

\section{Análisis de Resultados.}

\section{Naturaleza del Fluido}

El sabajón es un fluido que está conformado por materiales potencialmente electrorreológicos, tales como: proteínas, alcoholes y carbohidratos, moléculas polares que pueden ser orientadas por un campo eléctrico producido mediante la aplicación de un voltaje, lo cual puede observarse en los cambios reológicos presentados en el fluido durante las prácticas realizadas.

\section{Características Reológicas y el Tiempo}

De los resultados de la tabla 3, se observa que al variar el gradiente de velocidad tanto en forma ascendente como descendente, el índice de flujo n, no presenta variación significativa $(0,803-0,828)$, es decir, las características seudoplásticas permanecen durante el tiempo transcurrido entre las determinaciones con aumento y disminución de 
velocidad. Ahora bien, al graficar esfuerzo de corte $\tau$, contra gradiente de velocidad $\gamma$, en forma ascendente y luego en forma descendente, se encontró que la diferencia entre las dos áreas es mínima, correspondiendo a un grado de tixotropía. de 1,90\%. Estos dos antecedentes permiten afirmar que las propiedades reológicas del sabajón son independientes del tiempo, no clasificándose ni como reopéctico, ni como tixotrópico.

De acuerdo con los resultados obtenidos para el índice de flujo, n, el sabajón es un fluído ligeramente seudoplástico, con índice de flujo promedio de 0,815 y al ser sometido a diferentes voltajes y tiempos de aplicación, dicho índice disminuyó hasta 0,754, haciéndose más seudoplástico. De acuerdo con esta característica, su comportamiento se acomoda al modelo de ley de potencia.

\section{Análisis de Varianza}

Para determinar la significación de los resultados y encontrar los modelos matemáticos adecuados que den respuesta a la correlación de las variables, se realizaron análisis de regresión múltiple para cada una de las variables dependientes con respecto a las independientes, utilizado el software Statgraphics plus 5.0.

Ajuste del modelo de esfuerzo de corte contra tiempo y voltaje. Para este estudio se tomaron los datos de la tabla 2, realizándose un análisis de varianza para cada gradiente de velocidad y al considerar inicialmente para dichos análisis la variable dependiente esfuerzo de corte y las independientes voltaje y tiempo, se encontró que el tiempo no tiene una relación estadísticamente significativa con el esfuerzo de corte, ya que p para cada caso arrojó un valor $>0.1$ mostrando que el nivel de confianza es $<90 \%$. Al eliminar la variable t y realizar nuevamente el análisis, se encontraron resultados significativos, los cuales se muestran en las tablas de ANOVA presentadas a continuación.

Tabla 5. Análisis de regresión múltiple para esfuerzo de corte en función de voltaje y tiempo, para cada gradiente de velocidad

$\gamma=43,21 / \mathrm{s}$

Multiple Regression Analysis

Dependent variable: Esfuerzo de corte

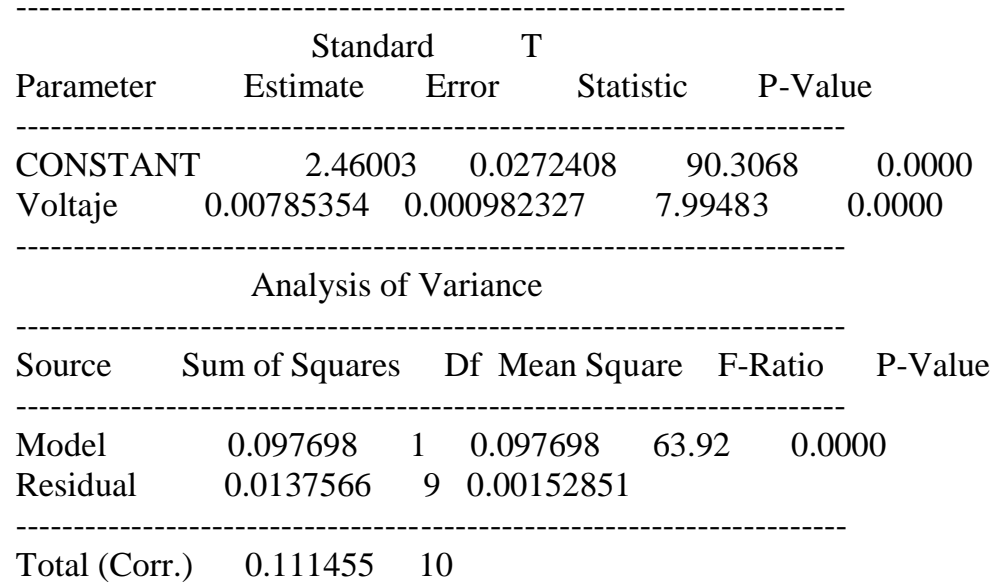


R-squared $=87.6572$ percent

R-squared (adjusted for d.f.) $=86.2858$ percent

Standard Error of Est. $=0.0390961$

Mean absolute error $=0.0294215$

Durbin-Watson statistic $=2.01065$

$\mathrm{F}_{1 \%, 1,9}=10,56$

De acuerdo con los resultados mostrados en la tabla 5, para $\boldsymbol{\gamma}=\mathbf{4 3 , 2} 1 / \mathbf{s}$, se encuentra que existe relación estadísticamente significativa entre la variable independiente voltaje con la variable dependiente esfuerzo de corte, con un nivel de confianza mayor del 99\% ( $\mathrm{P}<0.01$ y el Factor de ajuste $\left.\mathrm{F}_{\text {calculada }}>\mathrm{F}_{99 \text { tabulada }}\right)$.

En consecuencia el modelo matemático que representa esta relación se expresa en la siguiente ecuación:

$$
\tau=2,46003+0,00785354 \mathrm{v} \quad(\text { Ec. } 6)
$$

El R cuadrado estadístico en este modelo ajustado, indica el $87.29 \%$ de la variabilidad en el esfuerzo de corte. El R cuadrado ajustado, el cual es más conveniente para comparar modelos con diferente número de variables independientes, reporta un $86,29 \%$ de variabilidad. El error estándar de los estimados muestra que la desviación estándar de los residuos es de 0,0391. El error absoluto de la media, corresponde al valor promedio de los residuos y tiene un valor de 0,0294. En cuanto el valor de Durbin-Watson estadístico $>1,4$, muestra que probablemente no existe autocorrelación seria entre los residuos.

$\gamma=75,61 / \mathrm{s}$

Multiple Regression Analysis

Dependent variable: Esfuerzo de corte

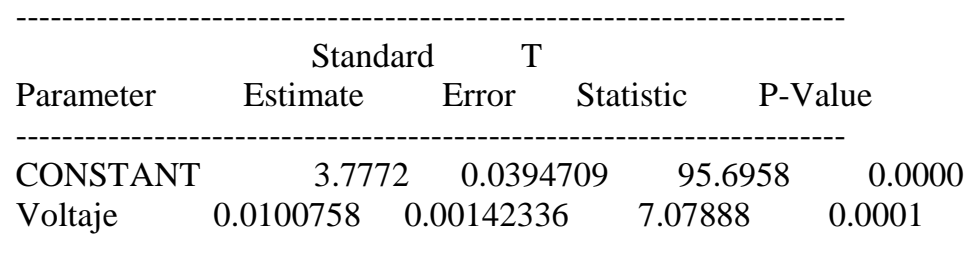

Analysis of Variance

Source Sum of Squares Df Mean Square F-Ratio P-Value

$\begin{array}{lccccc}\text { Model } & 60809 & 1 & 0.160809 & 50.11 & 0.0001 \\ \text { Residual } & 0.0288818 & 9 & 0.00320909 & \end{array}$

Total (Corr.) $\quad 0.189691 \quad 10$

$\mathrm{R}$-squared $=84.7743$ percent

R-squared (adjusted for d.f.) $=83.0825$ percent

Standard Error of Est. $=0.0566488$ 
Mean absolute error $=0.047989$

Durbin-Watson statistic $=2.30121$

$\mathrm{F}_{1 \%, 1,9}=10,56$

De acuerdo con los resultados mostrados en la tabla 5 , para $\boldsymbol{\gamma}=\mathbf{7 5 , 6} \mathbf{1 /}$, se encuentra que existe relación estadísticamente significativa entre la variable independiente voltaje con la variable dependiente esfuerzo de corte, con un nivel de confianza mayor del $99 \%\left(\mathrm{P}<0.01\right.$ y el Factor de ajuste $\mathrm{F}_{\text {calculada }}>\mathrm{F}_{99 \text { tabulada }}$ ).

En consecuencia el modelo matemático que representa esta relación se expresa en la siguiente ecuación:

$$
\tau=3,7772+0,0100758 \mathrm{v} \quad(\text { Ec. } 7)
$$

El R cuadrado estadístico en este modelo ajustado, indica el $87.77 \%$ de la variabilidad en el esfuerzo de corte. El R cuadrado ajustado, el cual es más conveniente para comparar modelos con diferente número de variables independientes, reporta un 83,08\% de variabilidad. El error estándar de los estimados muestra que la desviación estándar de los residuos es de 0,0566. El error absoluto de la media, corresponde al valor promedio de los residuos y tiene un valor de 0,0480. En cuanto el valor de DurbinWatson estadístico > 1,4, muestra que probablemente no existe autocorrelación seria entre los residuos.

$\gamma=124,21 / \mathrm{s}$

Multiple Regression Analysis

Dependent variable: Esfuerzo de corte

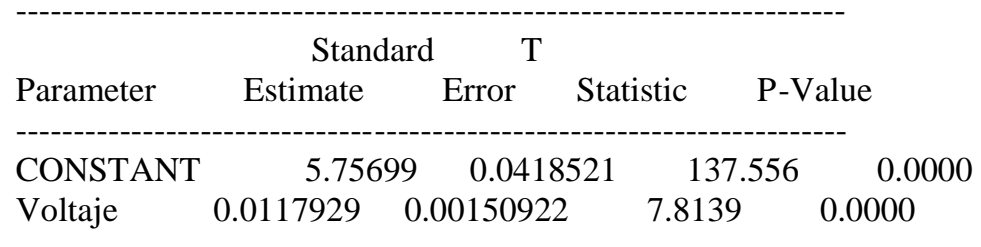

Analysis of Variance

Source Sum of Squares Df Mean Square F-Ratio P-Value

$\begin{array}{llllll}\text { Model } & 0.220292 & 1 & 0.220292 & 61.06 & 0.0000 \\ \text { Residual } & 0.0324717 & 9 & 0.00360797 & & \end{array}$

Total (Corr.) $\quad 0.252764 \quad 10$

$\mathrm{R}$-squared $=87.1533$ percent

R-squared (adjusted for d.f.) $=85.7259$ percent

Standard Error of Est. $=0.0600664$

Mean absolute error $=0.0484665$

Durbin-Watson statistic $=2.18723$

$\mathrm{F}_{1 \%, 1,9}=10,56$ 
De acuerdo con los resultados mostrados en la tabla 5, para $\boldsymbol{\gamma}=\mathbf{1 2 4 , 2} 1 / \mathbf{s}$, se encuentra que existe relación estadísticamente significativa entre la variable independiente voltaje con la variable dependiente esfuerzo de corte, con un nivel de confianza mayor del $99 \%$ $\left(\mathrm{P}<0.01\right.$ y el Factor de ajuste $\left.\mathrm{F}_{\text {calculada }}>\mathrm{F}_{99 \text { tabulada }}\right)$.

En consecuencia el modelo matemático que representa esta relación se expresa en la siguiente ecuación: $\tau=5,75699+0,0117929$ v $\quad$ (Ec. 8$)$

El R cuadrado estadístico en este modelo ajustado, indica el $87,15 \%$ de la variabilidad en el esfuerzo de corte. El R cuadrado ajustado, el cual es más conveniente para comparar modelos con diferente número de variables independientes, reporta un $85,73 \%$ de variabilidad. El error estándar de los estimados muestra que la desviación estándar de los residuos es de 0,0601. El error absoluto de la media, corresponde al valor promedio de los residuos y tiene un valor de 0,0485. En cuanto el valor de Durbin-Watson estadístico $>1,4$, muestra que probablemente no existe autocorrelación seria entre los residuos.

$\gamma=207,91 / \mathrm{s}$

Multiple Regression Analysis

Dependent variable: Esfuerzo de corte

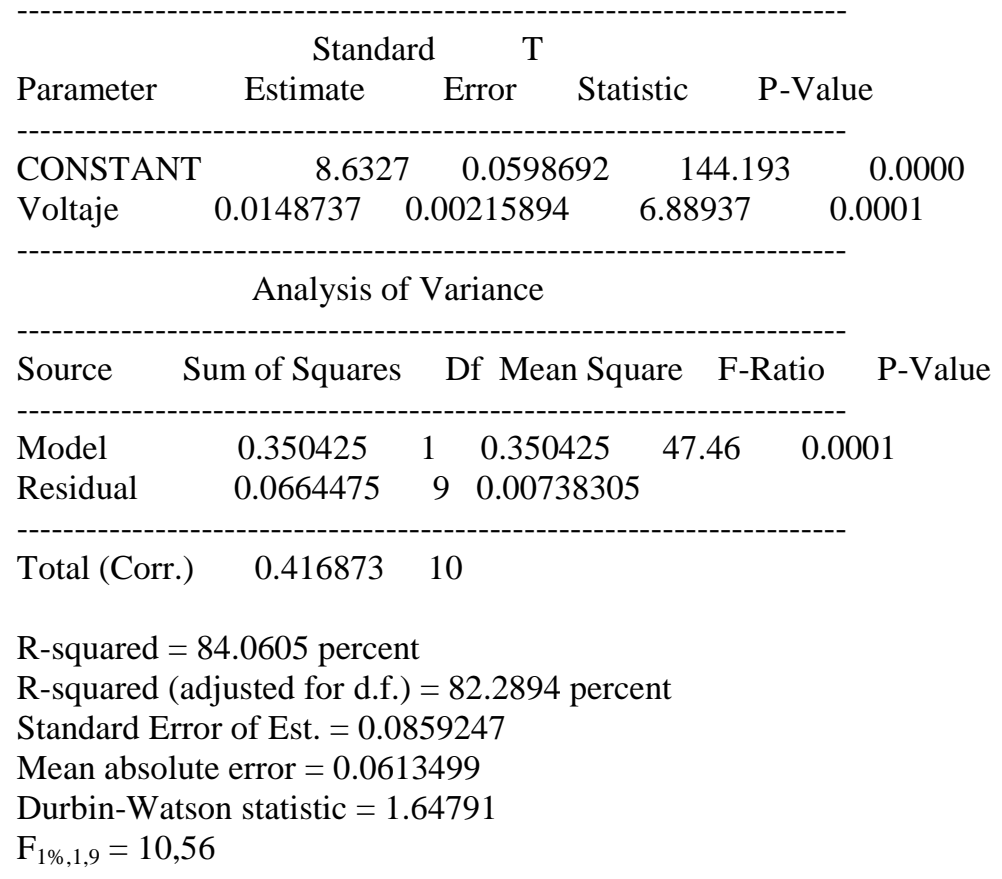

De acuerdo con los resultados mostrados en la tabla 5, para $\boldsymbol{\gamma}=\mathbf{2 0 7 , 9} \mathbf{1} / \mathbf{s}$, se encuentra que existe relación estadísticamente significativa entre la variable independiente voltaje con la variable dependiente esfuerzo de corte, con un nivel de confianza mayor del $99 \%$ $\left(\mathrm{P}<0.01\right.$ y el Factor de ajuste $\left.\mathrm{F}_{\text {calculada }}>\mathrm{F}_{99 \text { tabulada }}\right)$. 
En consecuencia el modelo matemático que representa esta relación se expresa en la siguiente ecuación: $\tau=8,6327+0,0148737$ v $\quad$ (Ec. 9)

El R cuadrado estadístico en este modelo ajustado, indica el 84,06\% de la variabilidad en el esfuerzo de corte. El R cuadrado ajustado, el cual es más conveniente para comparar modelos con diferente número de variables independientes, reporta un 82,29\% de variabilidad. El error estándar de los estimados muestra que la desviación estándar de los residuos es de 0,0859. El error absoluto de la media, corresponde al valor promedio de los residuos y tiene un valor de 0,0613. En cuanto el valor de Durbin-Watson estadístico $>1,4$, muestra que probablemente no existe autocorrelación seria entre los residuos.

$\gamma=348,31 / \mathrm{s}$

Multiple Regression Analysis

Dependent variable: Esfuerzo de corte

\begin{tabular}{|c|c|c|c|c|c|}
\hline \multirow[b]{2}{*}{ Parameter } & Standard & \multicolumn{2}{|c|}{$\mathrm{T}$} & \\
\hline & Estimate & Error & Stat & istic & Value \\
\hline CONSTANT & 12.8811 & 10.08 & 0202 & 158.986 & 0.0000 \\
\hline Voltaje & $0.0189394 \quad 0$ & 0.00292 & & 6.4824 & 0.0001 \\
\hline
\end{tabular}

\begin{tabular}{lccccc} 
Source & Sum of Squares & Df & Mean Square & F-Ratio & P-V \\
\hdashline Model & 0.568182 & 1 & 0.568182 & 42.02 & 0.0001 \\
Residual & 0.121691 & 9 & 0.0135212 & &
\end{tabular}

Total (Corr.) $\quad 0.689873 \quad 10$

R-squared $=82.3604$ percent

R-squared (adjusted for d.f.) $=80.4004$ percent

Standard Error of Est. $=0.116281$

Mean absolute error $=0.0704683$

Durbin-Watson statistic $=1.51195$

$\mathrm{F}_{1 \%, 1,9}=10,56$

De acuerdo con los resultados mostrados en la tabla 5, para $\boldsymbol{\gamma}=\mathbf{3 4 8 , 3} 1 / \mathbf{s}$, se encuentra que existe relación estadísticamente significativa entre la variable independiente voltaje con la variable dependiente esfuerzo de corte, con un nivel de confianza mayor del $99 \%$ $\left(\mathrm{P}<0.01\right.$ y el Factor de ajuste $\left.\mathrm{F}_{\text {calculada }}>\mathrm{F}_{99 \text { tabulada }}\right)$.

En consecuencia el modelo matemático que representa esta relación se expresa en la siguiente ecuación: $\tau=12,8811+0,0189394 \mathrm{v} \quad$ (Ec. 10) 
El R cuadrado estadístico en este modelo ajustado, indica el $82,36 \%$ de la variabilidad en el esfuerzo de corte. El R cuadrado ajustado, el cual es más conveniente para comparar modelos con diferente número de variables independientes, reporta un $80,40 \%$ de variabilidad. El error estándar de los estimados muestra que la desviación estándar de los residuos es de 0,1163 . El error absoluto de la media, corresponde al valor promedio de los residuos y tiene un valor de 0,0705. En cuanto el valor de Durbin-Watson estadístico $>1,4$, muestra que probablemente no existe autocorrelación seria entre los residuos.

$\gamma=580,51 / \mathrm{s}$

Multiple Regression Analysis

Dependent variable: Esfuerzo de corte

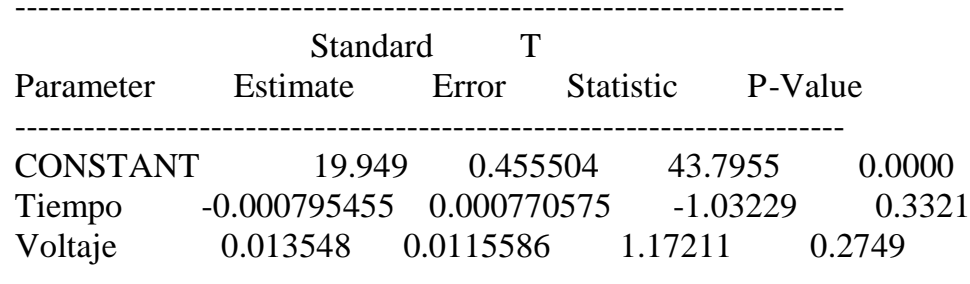

Analysis of Variance

Source Sum of Squares Df Mean Square F-Ratio P-Value

$\begin{array}{lccccc}\text { Model } & 0.516251 & 2 & 0.258126 & 1.22 & 0.3449 \\ \text { Residual } & 1.693 & 8 & 0.211625 & & \end{array}$

Total (Corr.) $\quad 2.20925 \quad 10$

R-squared (adjusted for d.f.) $=4.20957$ percent

Standard Error of Est. $=0.460028$

Mean absolute error $=0.315051$

Durbin-Watson statistic $=1.01177$

$\mathrm{F}_{1 \%, 1,9}=10,56$

Como puede verse en esta tabla de ANOVA, los valores de $\mathrm{P}$ tanto para el modelo como para cada una de las variables independientes, muestran que este modelo no tiene ningún significado estadístico. De igual forma los valores de F, R cuadrado y Durbin Watson, corroboran lo inferido anteriormente.

\section{Ajuste del modelo de viscosidad aparente contra voltaje y tiempo.}

Para este estudio se tomaron los datos de la tabla 2, realizándose un análisis de varianza para cada gradiente de velocidad y al considerar inicialmente para dichos análisis la variable dependiente viscosidad aparente y las independientes voltaje y tiempo, se encontró que el tiempo no tiene una relación estadísticamente significativa con la viscosidad aparente, ya que p para cada caso arrojó un valor $>0.1$ mostrando que el 
nivel de confianza es $<90 \%$. Al eliminar la variable t y realizar nuevamente el análisis, se encontraron resultados significativos, los cuales se muestran en las tablas de ANOVA presentadas a continuación.

Tabla 6. Análisis de regresión múltiple para viscosidad aparente en función de voltaje y tiempo, para cada gradiente de velocidad

$\gamma=43,21 / \mathrm{s}$

Multiple Regression Analysis

Dependent variable: Viscosidad Aparente

\begin{tabular}{lcccc} 
& \multicolumn{2}{c}{ Standard } & & \\
Parameter & Estimate & Error & Statistic & P-Value \\
----------------------------------------- & \\
CONSTANT & 56.89 & 0.610978 & 93.113 & 0.0000 \\
Voltaje & 0.183141 & 0.0220324 & 8.31236 & 0.0000
\end{tabular}

Analysis of Variance

\begin{tabular}{lccccc} 
Source & Sum of Squares & Df Mean Square & F-Ratio \\
\hline Model & 53.1286 & 1 & 53.1286 & 69.10 & 0.0000 \\
Residual & 6.92026 & 9 & 0.768918 & &
\end{tabular}

Total (Corr. $\quad 60.0489 \quad 10$

R-squared $=88.4756$ percent

R-squared (adjusted for d.f.) $=87.1951$ percent

Standard Error of Est. $=0.87688$

Mean absolute error $=0.667587$

Durbin-Watson statistic $=2.00478$

$\mathrm{F}_{1 \%, 1,9}=10,56$

De acuerdo con los resultados mostrados en la tabla 6 , para $\boldsymbol{\gamma}=\mathbf{4 3 , 2} 1 / \mathbf{s}$, se encuentra que existe relación estadísticamente significativa entre la variable independiente voltaje con la variable dependiente viscosidad aparente, con un nivel de confianza mayor del 99\% ( $\mathrm{P}<0.01$ y el Factor de ajuste $\mathrm{F}_{\text {calculada }}>\mathrm{F}_{99 \text { tabulada }}$ ).

En consecuencia el modelo matemático que representa esta relación se expresa en la siguiente ecuación: $\eta=56,89+0,183141 \mathrm{v} \quad$ (Ec. 11)

El R cuadrado estadístico en este modelo ajustado, indica el $88,48 \%$ de la variabilidad de la viscosidad aparente. El R cuadrado ajustado, el cual es más conveniente para comparar modelos con diferente número de variables independientes, reporta un 87,20\% de variabilidad. El error estándar de los estimados muestra que la desviación estándar de los residuos es de 0,8769 . El error absoluto de la media, corresponde al valor promedio de los residuos y tiene un valor de 0,6676. En cuanto el valor de Durbin-Watson 
estadístico $>1,4$, muestra que probablemente no existe autocorrelación seria entre los residuos.

$\gamma=75,61 / \mathrm{s}$

Multiple Regression Analysis

Dependent variable: Viscosidad Aparente

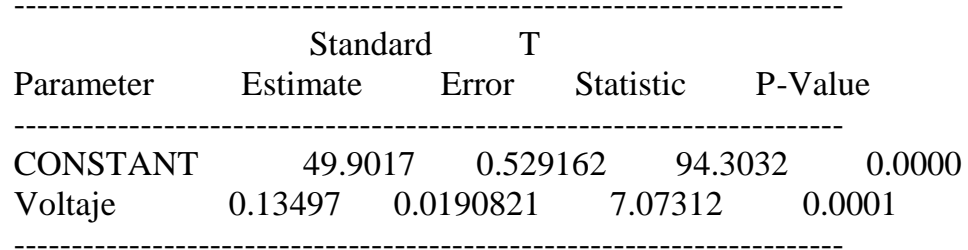

Analysis of Variance

Source Sum of Squares Df Mean Square F-Ratio P-Value

$\begin{array}{llllll}\text { Model } & 28.8554 & 1 & 28.8554 & 50.03 & 0.0001 \\ \text { Residual } & 5.19097 & 9 & 0.576774 & & \end{array}$

Total (Corr.) $\quad 34.0464 \quad 10$

R-squared $=84.7533$ percent

R-squared (adjusted for d.f.) $=83.0592$ percent

Standard Error of Est. $=0.759456$

Mean absolute error $=0.644127$

Durbin-Watson statistic $=2.27182$

$\mathrm{F}_{1 \%, 1,9}=10,56$

De acuerdo con los resultados mostrados en la tabla 6 , para $\boldsymbol{\gamma}=\mathbf{7 5 , 6} \mathbf{1 / s}, \quad$ se encuentra que existe relación estadísticamente significativa entre la variable independiente voltaje con la variable dependiente viscosidad aparente, con un nivel de confianza mayor del 99\% ( $\mathrm{P}<0.01$ y el Factor de ajuste $\mathrm{F}_{\text {calculada }}>\mathrm{F}_{99 \text { tabulada }}$ ).

En consecuencia el modelo matemático que representa esta relación se expresa en la siguiente ecuación: $\eta=49,9017+0,13497 \mathrm{v} \quad$ (Ec. 12)

El R cuadrado estadístico en este modelo ajustado, indica el $84,75 \%$ de la variabilidad de la viscosidad aparente. El R cuadrado ajustado, el cual es más conveniente para comparar modelos con diferente número de variables independientes, reporta un $83,06 \%$ de variabilidad. El error estándar de los estimados muestra que la desviación estándar de los residuos es de 0,7595 . El error absoluto de la media, corresponde al valor promedio de los residuos y tiene un valor de 0,6441. En cuanto el valor de Durbin-Watson estadístico $>1,4$, muestra que probablemente no existe autocorrelación seria entre los residuos. 
$\gamma=124,21 / \mathrm{s}$

Multiple Regression Analysis

Dependent variable: Viscosidad Aparente

\begin{tabular}{|c|c|c|c|c|c|c|}
\hline \multirow[b]{2}{*}{ Parameter } & Standard & $\mathrm{T}$ & \multirow{2}{*}{\multicolumn{2}{|c|}{ Statistic }} & \multirow{2}{*}{\multicolumn{2}{|c|}{ P-Value }} \\
\hline & Estimate & Error & & & & \\
\hline CONSTANT & 46.3665 & $\begin{array}{l}5 \quad 0.33 \\
\end{array}$ & 2752 & 13 & .343 & 0.0000 \\
\hline Voltaje & 0.0944583 & 0.011999 & & 7.871 & & 0.0000 \\
\hline
\end{tabular}

Analysis of Variance

\begin{tabular}{llllll}
\hline Source & Sum of Squares & Df Mean Square & F-Ratio \\
\hline Model & 14.133 & 1 & 14.133 & 61.97 & 0.0000 \\
Residual & 2.05263 & 9 & 0.22807 & &
\end{tabular}

Total (Corr.) $\quad 16.1857 \quad 10$

R-squared $=87.3182$ percent

R-squared (adjusted for d.f.) $=85.9091$ percent

Standard Error of Est. $=0.477567$

Mean absolute error $=0.383796$

Durbin-Watson statistic $=2.17378$

$\mathrm{F}_{1 \%, 1,9}=10,56$

De acuerdo con los resultados mostrados en la tabla 6 , para $\boldsymbol{\gamma}=\mathbf{1 2 4 , 2} 1 / \mathrm{s}$, se encuentra que existe relación estadísticamente significativa entre la variable independiente voltaje con la variable dependiente viscosidad aparente, con un nivel de confianza mayor del 99\% ( $\mathrm{P}<0.01$ y el Factor de ajuste $\mathrm{F}_{\text {calculada }}>\mathrm{F}_{99 \text { tabulada }}$ ).

En consecuencia el modelo matemático que representa esta relación se expresa en la siguiente ecuación: $\eta=46,3665+0,0944583$ v $\quad$ (Ec. 13)

El R cuadrado estadístico en este modelo ajustado, indica el $87,32 \%$ de la variabilidad de la viscosidad aparente. El R cuadrado ajustado, el cual es más conveniente para comparar modelos con diferente número de variables independientes, reporta un 85,91\% de variabilidad. El error estándar de los estimados muestra que la desviación estándar de los residuos es de 0,4776. El error absoluto de la media, corresponde al valor promedio de los residuos y tiene un valor de 0,3838. En cuanto el valor de Durbin-Watson estadístico > 1,4, muestra que probablemente no existe autocorrelación seria entre los residuos. 
$\gamma=207,91 / \mathrm{s}$

Multiple Regression Analysis

Dependent variable: Viscosidad Aparente

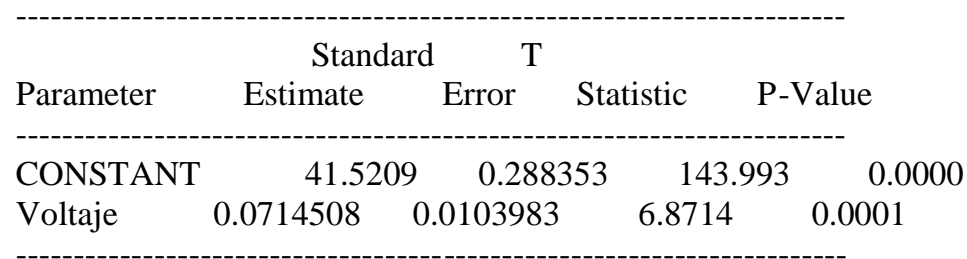

Analysis of Variance

Source Sum of Squares Df Mean Square F-Ratio P-Value

$\begin{array}{llllll}\text { Model } & 8.08665 & 1 & 8.08665 & 47.22 & 0.0001 \\ \text { Residual } & 1.54142 & 9 & 0.171269 & & \end{array}$

Total (Corr.) $\quad 9.62807 \quad 10$

R-squared $=83.9904$ percent

R-squared (adjusted for d.f.) $=82.2115$ percent

Standard Error of Est. $=0.413846$

Mean absolute error $=0.300079$

Durbin-Watson statistic $=1.69838$

$\mathrm{F}_{1 \%, 1,9}=10,56$

De acuerdo con los resultados mostrados en la tabla 6 , para $\boldsymbol{\gamma}=\mathbf{2 0 7 , 9} \mathbf{1} / \mathbf{s}$, se encuentra que existe relación estadísticamente significativa entre la variable independiente voltaje con la variable dependiente viscosidad aparente, con un nivel de confianza mayor del 99\% ( $\mathrm{P}<0.01$ y el Factor de ajuste $\left.\mathrm{F}_{\text {calculada }}>\mathrm{F}_{99 \text { tabulada }}\right)$.

En consecuencia el modelo matemático que representa esta relación se expresa en la siguiente ecuación: $\eta=41,5209+0,0714508$ v $\quad$ (Ec. 14)

El R cuadrado estadístico en este modelo ajustado, indica el $83,99 \%$ de la variabilidad de la viscosidad aparente. El R cuadrado ajustado, el cual es más conveniente para comparar modelos con diferente número de variables independientes, reporta un $82,21 \%$ de variabilidad. El error estándar de los estimados muestra que la desviación estándar de los residuos es de 0,4138. El error absoluto de la media, corresponde al valor promedio de los residuos y tiene un valor de 0,3001. En cuanto el valor de Durbin-Watson estadístico $>1,4$, muestra que probablemente no existe autocorrelación seria entre los residuos. 
$\gamma=348,31 / \mathrm{s}$

Multiple Regression Analysis

Dependent variable: Viscosidad Aparente

\begin{tabular}{|c|c|c|c|c|c|c|}
\hline \multirow[b]{2}{*}{ Parameter } & Standard & $\mathrm{T}$ & \multirow{2}{*}{\multicolumn{2}{|c|}{ Statistic }} & \multirow{2}{*}{\multicolumn{2}{|c|}{ P-Value }} \\
\hline & Estimate & Error & & & & \\
\hline CONSTANT & 36.9812 & $\begin{array}{ll}2 & 0.2\end{array}$ & 0023 & & .772 & 0.0000 \\
\hline Voltaje & $0.0544609 \quad 0$ & 0.00829 & & 6.565 & & 0.0001 \\
\hline
\end{tabular}

Analysis of Variance

\begin{tabular}{llllll} 
Source & Sum of Squares & Df Mean Square & F-Ratio P-Value \\
\hline Model & 4.69812 & 1 & 4.69812 & 43.11 & 0.0001 \\
Residual & 0.980876 & 9 & 0.108986 & &
\end{tabular}

Total (Corr.) $\quad 5.679 \quad 10$

R-squared $=82.728$ percent

R-squared (adjusted for d.f.) $=80.8089$ percent

Standard Error of Est. $=0.330131$

Mean absolute error $=0.199306$

Durbin-Watson statistic $=1.50569$

$\mathrm{F}_{1 \%, 1,9}=10,56$

De acuerdo con los resultados mostrados en la tabla 6 , para $\gamma=\mathbf{3 4 8 , 3} 1 / \mathrm{s}$, se encuentra que existe relación estadísticamente significativa entre la variable independiente voltaje con la variable dependiente viscosidad aparente, con un nivel de confianza mayor del 99\% ( $\mathrm{P}<0.01$ y el Factor de ajuste $\mathrm{F}_{\text {calculada }}>\mathrm{F}_{99 \text { tabulada }}$ ).

En consecuencia el modelo matemático que representa esta relación se expresa en la siguiente ecuación: $\eta=36,9812+0,0544609 v \quad$ (Ec. 15)

El R cuadrado estadístico en este modelo ajustado, indica el $82,73 \%$ de la variabilidad de la viscosidad aparente. El R cuadrado ajustado, el cual es más conveniente para comparar modelos con diferente número de variables independientes, reporta un 80,81\% de variabilidad. El error estándar de los estimados muestra que la desviación estándar de los residuos es de 0,3301. El error absoluto de la media, corresponde al valor promedio de los residuos y tiene un valor de 0,1993. En cuanto el valor de Durbin-Watson estadístico $>1,4$, muestra que probablemente no existe autocorrelación seria entre los residuos. 
$\gamma=580,51 / \mathrm{s}$

Multiple Regression Analysis

Dependent variable: Viscosidad Aparente

\begin{tabular}{|c|c|c|c|c|c|c|}
\hline \multirow[b]{2}{*}{ Parameter } & Standard & $\mathrm{T}$ & \multirow{2}{*}{\multicolumn{2}{|c|}{ Statistic }} & \multirow{2}{*}{\multicolumn{2}{|c|}{ P-Value }} \\
\hline & Estimate & Error & & & & \\
\hline CONSTANT & 34.5474 & 0.81 & 6188 & & 3277 & 0.0000 \\
\hline Voltaje & $0.0233813 \quad 0$ & 0.020711 & & 1.128 & & 0.2916 \\
\hline Tiempo & -0.0013729 & 0.00138 & & -0.99 & 315 & 0.3492 \\
\hline
\end{tabular}

Analysis of Variance

\begin{tabular}{llllll}
\hline Source & Sum of Squares & Df Mean Square & F-Ratio \\
\hline Model & 1.53771 & 2 & 0.768854 & 1.13 & 0.3692 \\
Residual & 5.43569 & 8 & 0.679461 & &
\end{tabular}

Total (Corr.) $\quad 6.97339 \quad 10$

$\mathrm{R}$-squared $=22.0511$ percent

R-squared (adjusted for d.f.) $=2.56384$ percent

Standard Error of Est. $=0.824294$

Mean absolute error $=0.573792$

Durbin-Watson statistic $=2.05261$

$\mathrm{F}_{1 \%, 1,9}=10,56$

Como puede verse en esta tabla de ANOVA, los valores de P tanto para el modelo como para cada una de las variables independientes, muestran que este modelo no tiene ningún significado estadístico. De igual forma los valores de $\mathrm{F}$ y $\mathrm{R}$ cuadrado corroboran lo inferido anteriormente.

\section{Ajuste del modelo de índice de consistencia en función del voltaje y del tiempo.}

Para este caso se encontró que el modelo más conveniente depende de las variables Ln voltaje y Ln tiempo. Los datos de este análisis se encuentran en la tabla 7.

Tabla 7. Análisis de regresión múltiple para Índice de Consistencia en función de voltaje y tiempo

Multiple Regression Analysis

Dependent variable: Indice de Consistencia

Parameter Estimate Error Statistic P-Value




$\begin{array}{lrccr}\text { Ln Voltaje } & 0.0697963 & 0.00591153 & 11.8068 & 0.0000 \\ \text { Voltaje } & -0.00286415 & 0.000665577 & -4.30325 & 0.0020\end{array}$

Analysis of Variance

Source Sum of Squares Df Mean Square F-Ratio P-Value

\begin{tabular}{|c|c|c|c|c|}
\hline Model & 0.22385 & 2 & $0.111925 \quad 449.61$ & 0.000 \\
\hline Residual & 0.00224043 & 9 & 0.000248936 & \\
\hline
\end{tabular}

Total (Corr.) $\quad 0.22609 \quad 11$

R-squared $=99.01$ percent

R-squared (adjusted for d.f.) $=98.90$ percent

Standard Error of Est. $=0.0157777$

Mean absolute error $=0.0100446$

Durbin-Watson statistic $=2.90022$

$\mathrm{F}_{1 \%, 1,9}=8,02$

De acuerdo con los datos de la tabla 7, se encuentra que existe una relación estadísticamente significativa entre las variables independientes ln voltaje y voltaje, con la variable dependiente índice de consistencia, con un nivel de confianza mayor del $99 \%$ ( $\mathrm{P}<0.01$ y $\mathrm{F}_{\text {calculada }}>\mathrm{F}_{99 \text { tabulada }}$ ).

En consecuencia el modelo matemático que representa esta relación se expresa en la siguiente ecuación: $\mathrm{k}=0,0697963 \ln \mathrm{v}-0,00286415 \mathrm{v} \quad$ (Ec. 16)

El R cuadrado estadístico en este modelo ajustado, indica el 99,01\% de la variabilidad en el índice de consistencia. El R cuadrado ajustado, reporta un 98,90 \% de variabilidad. El error estándar de los estimados muestra que la desviación estándar de los residuos es de 0,01778. El error absoluto de la media, correspondiente al valor promedio de los residuos tiene un valor de 0,01004 .

Ajuste del modelo de índice de flujo vs voltaje y tiempo. Para este caso se encontró que el modelo matemático más conveniente no depende del tiempo, sino de las variables voltaje y Ln voltaje. Los datos de este análisis se muestran en la table 8.

Tabla 8. Análisis de regresión múltiple para Índice de Flujo función de voltaje y tiempo

Multiple Regression Analysis

Dependent variable: Indice de Flujo

\begin{tabular}{|c|c|c|c|c|}
\hline \multirow[b]{2}{*}{ Parameter } & \multicolumn{2}{|c|}{ Standard } & $\mathrm{T}$ & \multirow[b]{2}{*}{ P-Value } \\
\hline & Estimate & Error & Statistic & \\
\hline Ln Voltaje & 0.398377 & 0.033772 & 11.796 & 0.0000 \\
\hline Voltaje & -0.0182839 & 0.00380239 & -4.80853 & 0.0010 \\
\hline
\end{tabular}


Analysis of Variance

Source Sum of Squares Df Mean Square F-Ratio P-Value

$\begin{array}{llllll}\text { Model } & 6.4417 & 2 & 3.22008 & 396.34 & 0.0000 \\ \text { Residual } & 0.0731218 & 9 & 0.00812465 & \end{array}$

Total (Corr.) $\quad 6.51329 \quad 11$

R-squared $=98.8773$ percent

R-squared (adjusted for d.f.) $=98.7526$ percent

Standard Error of Est. $=0.0563523$

Mean absolute error $=0.0666462$

Durbin-Watson statistic $=2.67497$

$\mathrm{F}_{1 \%, 1,9}=8,02$

De acuerdo con los datos de la tabla 8, se encuentra que existe una relación estadísticamente significativa entre las variables independientes, voltaje y ln voltaje, con la variable dependiente índice de flujo, con un nivel de confianza mayor del $99 \%$ (P < 0.01 y $\mathrm{F}_{\text {calculada }}>\mathrm{F}_{99 \text { tabulada }}$ ). En consecuencia el modelo matemático que representa esta relación se expresa en la siguiente ecuación: $\eta=0,398377 \operatorname{lnv}-0,0182839 v \quad$ (Ec. 17)

El R cuadrado estadístico en este modelo ajustado, indica el 98,88\% de la variabilidad en el índice de flujo. El R cuadrado ajustado, reporta un 98,75\% de variabilidad. El error estándar de los estimados muestra que la desviación estándar de los residuos es de 0,05635. El error absoluto de la media, correspondiente al valor promedio de los residuos tiene un valor de 0,002372

\section{Conclusiones}

Los modelos determinados mediante las prácticas realizadas, permiten obtener las siguientes conclusiones:

- Las características reológicas del sabajón (sin aplicación de voltaje) son independientes del tiempo, ya que el grado de tixotropía es de 1,90\%, valor relativamente bajo, como puede observarse en la figura 3, lo cual puede responder al error experimental y no al cambio de las características reológicas en función del tiempo.

- La muestra de sabajón estudiada, es un fluido ligeramente seudoplástico, como lo indica el valor medio de índice de flujo de 0,816 .

- El índice de flujo, n, disminuye con la aplicación de voltaje desde 0,815 hasta 0,754, lo que permite concluir que la seudoplasticidad del sabajón aumenta ligeramente con el tratamiento eléctrico.

- El índice de consistencia, k, aumenta con la aplicación de voltaje desde 0,112 hasta 0,155 , permitiendo inferir que la viscosidad del fluído (sabajón) aumenta debido a mejor ordenamiento molecular de la muestra tratada.

- El comportamiento reológico del sabajón, con y sin aplicación de voltaje, se ajusta al modelo de la ley de potencia, presentando un alto grado de correlación entre el 
esfuerzo de corte y el gradiente de velocidad, como lo muestran las curvas de flujo anexas.

- El esfuerzo de corte presenta una correlación estadísticamente significativa con el voltaje para valores de gradiente de velocidad entre 43,2 y 348,3 1/s, no mostrando el mismo comportamiento para el valor de 580,5 1/s, como se aprecia en la tabla 7.

- La viscosidad aparente presenta una correlación estadísticamente significativa con el voltaje, para valores de gradiente de velocidad entre 43,2 y 348,3 1/s, no mostrando el mismo comportamiento para el valor de 580,5 1/s, como se aprecia en la tabla 8.

- Hay una correlación estadísticamente significativa entre el índice de consistencia y las variables independientes Ln voltaje y voltaje, como se observa en la tabla 9.

- Hay una correlación estadísticamente significativa entre el índice de flujo y las variables independientes voltaje y ln voltaje, lo cual puede observarse en la tabla 10.

- El tiempo no es una variable significativa que influya sobre las características reológicas del sabajón en el intervalo de voltaje aplicado.

\section{Bibliografía}

[d Barros Neto, B. 2001. Como Fazer experimentos: pesquisa e desenvolvimento na ciencia en la industria. Editora UNICAMP, Campinas, Brazil.

[D] Coulson, J. M. Y Richarson J. R. 1979. Ingeniería Química. Editorial.

[D] Reverté S.A. Barcelona.

[D] Daubert, C.R., Steffe, J.F. 1997. Electrorheological behavior of milk chocolate. Journal of textura Studies, 27 (1) 93-108.

[D] Daubert, C.R. 1997. Electhorheology of fluid foods. Dissertation Abstracts International, B: 57 (9) 5406 order no. DA9706474,191pp.

[Da] Daubert, C.R., Steffe, J.F., Srivastava, A. 1998. Predicting the electrorheological behavior of milk chocolate. Journal of Food Process Engineering, 21 (3) 249-261.

[Dd Horn, R. 2000. The effect of electric fields on thin liquid films. www.iwri.city.unisa.edu.au/projects.

[C] Kann, R.C. y Montgomery S. 1998. Enhanced Electrorheological fluids using anisotropic particles. Journal of Rheology, 42 (3).

[C] Larson, R. G. 1999. The structure and rheology of complex fluids. Oxford University Press. New York.

[D] Morrison F. A. 2001. Understanding rheology. Oxford University Press. New York.

[D] Muller H. G. 1973. Introducción a la reología de los alimentos. Editorial Acribia. Zaragoza (España).

[C] Perry J. H. 1950. Chemical Engineers's Handbook. 1950. McGraw- Hill Book Company. Inc USA.

[C] Sotelo I. 2001. Seminario de Investigación. UNAD. Bogotá, Colombia.

[D] Steffe, J.F. 1996. Rheological Methods in Food Process Engineering. Segunda Edición. Freeman Press. US.

[D] www.adepta.Com/Innovacionestecnológicas/PulsosEléctricos (activo en Septiembre 2002).

[D] Zumalacárregui. L. 2001. Notas de aula sobre reología. Instituto Superior Politécnico José Antonio Echeverría. La Habana, Cuba. 